\title{
Correction to: Lost in Translation? On the Need for Convergence in Animal and Human Studies on the Role of Dopamine in Diet-Induced Obesity
}

\author{
Lieneke K. Janssen ${ }^{1,2}$ - Nadine Herzog ${ }^{1,2}$ - Maria Waltmann ${ }^{1,2}$ - Nora Breuer ${ }^{1,2}$. Kathleen Wiencke ${ }^{1,2}$. \\ Franziska Rausch ${ }^{1,2,3}$ • Hendrik Hartmann ${ }^{2,3}$ - Maria Poessel ${ }^{2}$ - Annette Horstmann 1,2,3,4
}

Published online: 14 September 2019

(C) Springer Nature Switzerland AG 2019

\section{Correction to: Current Addiction Reports https://doi.org/10.1007/s40429-019-00268-w}

The original article unfortunately contained a mistake. In the online version of the paper, the entire Box 1 was missing, and the labels of the 2 tables were swapped.

The original version has been corrected.

Publisher's Note Springer Nature remains neutral with regard to jurisdictional claims in published maps and institutional affiliations.

The online version of the original article can be found at https://doi.org/ 10.1007/s40429-019-00268-w

Annette Horstmann

horstmann@cbs.mpg.de

1 Integrated Research and Treatment Center Adiposity Diseases, Leipzig University Medical Center, Leipzig, Germany

2 Department of Neurology, Max Planck Institute for Human Cognitive and Brain Sciences, Leipzig, Germany

3 Collaborative Research Centre 1052 “Obesity Mechanisms”, Leipzig University Medical Center, Leipzig, Germany

4 Department of Psychology and Logopedics, Faculty of Medicine, University of Helsinki, Helsinki, Finland 